
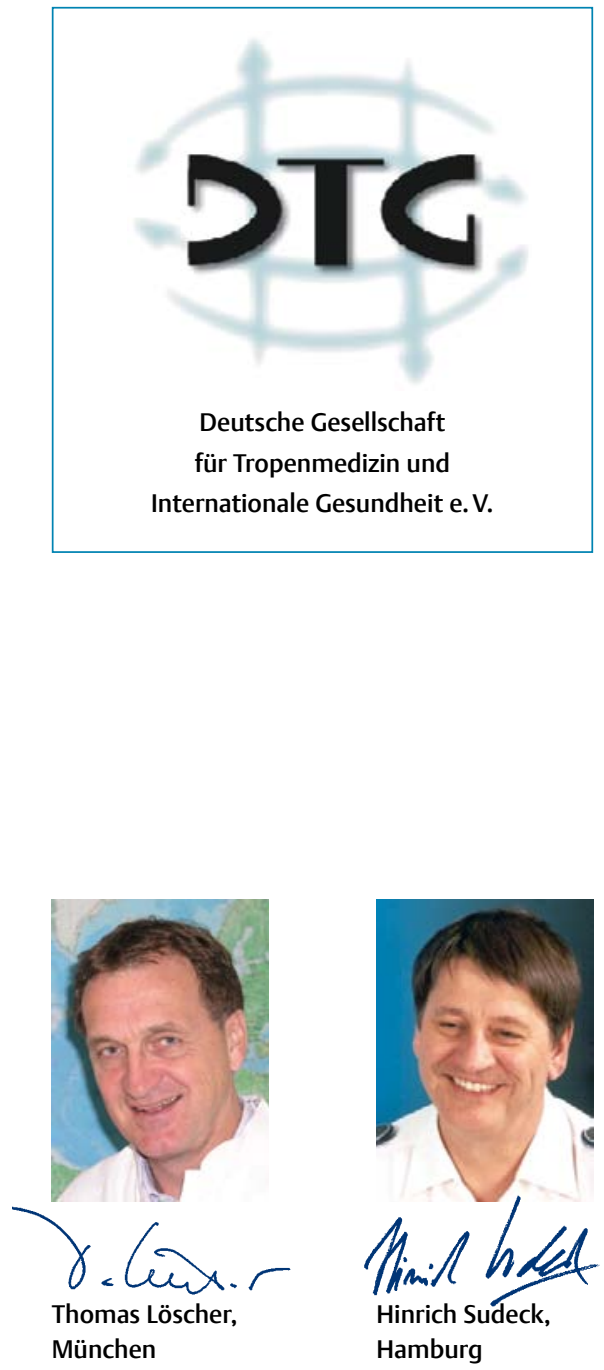

München Hamburg

\title{
Liebe Mitglieder der DTG,
}

wenn dieses Heft erscheint, steht die DTG-Mitgliederversammlung und Jahrestagung unserer Fachgesellschaft vom 14. bis 17. März 2012 in Heidelberg kurz bevor. Die DTG hat einen neuen Vorstand gewählt, der dann sein Amt antreten wird. Leider können wir Ihnen nicht schon in diesem Heft über das Ergebnis der Vorstandswahl berichten, das zum Druckschluss noch nicht vorliegt. Die Wahlbeteiligung ist aber befriedigend bis gut und wir haben auch schon eine Zahl neuer EMail-Adressen von Ihnen erhalten - dafür bedanken wir uns und hoffen, dass wir irgendwann einmal Sie alle elektronisch erreichen können. Das würde sehr viel an Portokosten sparen und eine noch größere Aktualität unserer Informationen erlauben. Ich erhoffe mir weiterhin von Ihnen Rückmeldungen zu Zahl und Art der versendeten Materialien, Anzeigen und Kongressankündigungen und ob Sie mehr oder weniger wollen - oder eher Anderes? Innerhalb des Vorstands haben wir auch diskutiert, ob die DTG Mitglied einer AG Infektiologie (AG-INF) werden sollte, die sich aus verschiedenen Fachgesellschaften heraus begründen wird, um ein abgestimmtes und gemeinsames Vorgehen bei infektiologischen Fragen oder öffentlichen Stellungnahmen zu ermöglichen.
Programm und Zweck der AG finden Sie auf der folgenden Seite.

Im Vorstand haben wir 2 neue Mitglieder, die niedergelassene Tropenmediziner sind - Dr. Erik Krause aus Freiburg und Dr. Fritz Holst aus Marburg, die sich hier vorstellen. Im Vorstand sind sie für den Aufbau einer Plattform für Niedergelassene innerhalb der DTG verantwortlich. Auf der Jahrestagung gibt es eine wichtige Veranstaltung zu diesem Thema: Wir laden Sie am Freitag, den 16. März 2012, um 16 Uhr, zur Veranstaltung „Tropenmedizin an der Schnittstelle zur Praxis - Quo vadis?“ ein. Themen sind die Belange niedergelassener tropenmedizinisch tätiger Ärzte. Ziele sind: Bestandsaufnahme der Situation niedergelassener Tropenmedizin, Erstellung eines Forderungskatalogs und Vorbereitung einer Internetplattform der DTG, die den Dialog innerhalb der niedergelassenen Tropenärzteschaft sowie mit den Tropeninstituten erleichtern soll. Leider haben wir auch wieder den Tod eines wichtigen und langjährigen Mitglieds zu betrauern: Aart van Soest ist im Alter von 83 Jahren verstorben. Bitte lesen Sie den Nachruf von Hans Jochen Diesfeld auf diesen Seiten.

Aus Hamburg und München grüßen Sie herzlich Hinrich Sudeck und Thomas Löscher

\section{Nachruf \\ Aart Hendrik van Soest (1928-2011)}

Am 30. November 2011 verstarb Aart Hendrik van Soest, kurz nachdem seine Frau Ineke von ihm gegangen war.

Aart van Soest war ein leidenschaftlicher Arzt und begnadeter Lehrer. Seine berufliche Vita wird sicher vom Deutschen Institut für Ärztliche Mission (DIFÄM) gewürdigt werden, dem er Jahrzehnte angehörte und dessen Geist und Auftrag er, so wie es ein Außenstehender erkennen konnte, wesentlich und nachhaltig mitgeprägt hat.

„Wir Heidelberger“, „Generationen“ von „Schülern“, die Studenten und Teilnehmer unserer „Entwicklungshelferkurse“ (EH-Kurs) ab 1974, wir Dozenten und Referenten und ganz persönlich ich haben ihm unendlich viel zu verdanken.

Seine äußerst kritische Sichtweise einer „Medizin in Entwicklungsländern“, wie es seinerzeit hieß, unsere Rolle hierin hat uns vor allem bei der Konzeption und Durchführung des EH-Kurses ganz wesentlich die Augen geöffnet und diesen mitgeprägt.

Aart van Soest war mir persönlich ein Leitbild nicht nur als Kenner der Probleme mit denen wir uns befassten. Auch als Arzt und Tropenmediziner war er für uns in den ersten Jahren des Aufbaus des Instituts ein kompetenter Berater.

Er hat uns die Augen geöffnet für Geburt und Tod, Gesundheit und Krankheit, Heilung und Medizin als kulturelles System, unsere eigene Kultur, aus der die „Schulmedizin“ entstanden ist, mit einbezogen.

Er setzte und verteidigte Maßstäbe, vor allem auch Wertmaßstäbe, was gerade in der Zeit seines Engagements bei uns von großer Wichtigkeit war.

Man kann heute sagen, dass diejenigen, die an seinem Denken teilhaben durften, denen er Lehrer war, ihm wesentliche Impulse für die eigene Arbeit und auch Sichtweise verdanken. Als seinerzeitiger Begründer des „EH-Kurses“ möchte ich dies mit großer Dankbarkeit und tiefem Respekt in Erinnerung bringen. Er hat uns sehr viel gegeben.

Hans Jochen Diesfeld, Starnberg 


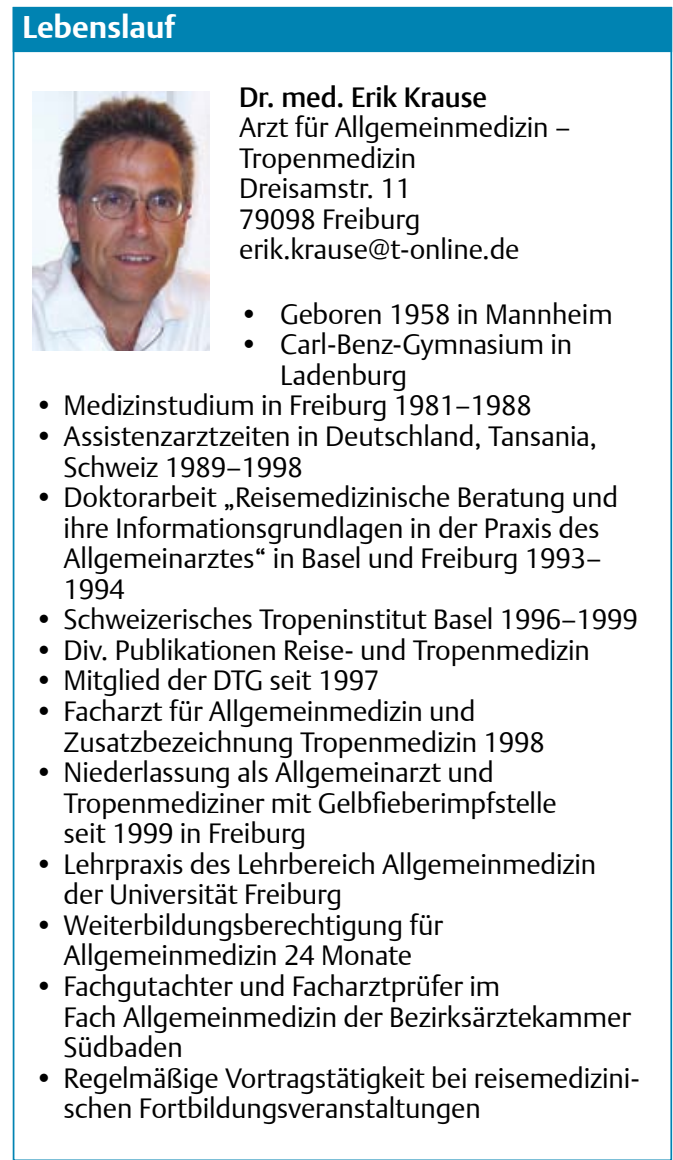

\begin{tabular}{|c|c|}
\hline \multicolumn{2}{|l|}{ Lebenslauf } \\
\hline$(4)$ & $\begin{array}{l}\text { Dr. med. Fritz Holst } \\
\text { Internist, Tropenmediziner, Hämatologe/Onkologe, Infektiologe } \\
\text { Lehrbeauftragter der Philipps-Universität Marburg } \\
\text { Tropen- u. Reisemedizinisches Zentrum Marburg } \\
\text { Liebigstr. 21, } 35037 \text { Marburg, holst@staff.uni-marburg.de }\end{array}$ \\
\hline & $\begin{array}{l}\text { Hochschulstudium } \\
\text { - 1976-1982 Humanmedizin an der Philipps-Universität Marburg } \\
\text { - 1992-1993 Studium der Orientalistik an der Universität Hamburg }\end{array}$ \\
\hline \multicolumn{2}{|c|}{$\begin{array}{l}\text { Weiterbildung } \\
\text { - Fachgebietsbezeichnung Innere Medizin } 1990 \\
\text { - Zusatzbezeichnung Tropenmedizin } 1994 \\
\text { - Zusatzbezeichnung Hämatologie/ Internistische Onkologie } 1996 \\
\text { - Zusatzbezeichnung Infektiologe } 2006\end{array}$} \\
\hline \multicolumn{2}{|c|}{$\begin{array}{l}\text { Promotion } \\
\text { - } 1986 \text { Thema: Proteolyse von Gerinnungsfaktor XIII durch lysosomale Enzyme } \\
\text { neutrophiler Granulozyten }\end{array}$} \\
\hline \multicolumn{2}{|c|}{$\begin{array}{l}\text { Klinische Tätigkeit } \\
\text { - Zentrum Innere Medizin, Philipps-Universität Marburg 1983-84 } \\
\text { - Prosper-Hospital, Recklinghausen 1984-88 } \\
\text { - Bernhard-Nocht-Institut, Hamburg 1988-90 } \\
\text { - Allgemeines Krankenhaus St. Georg, Hamburg 1990-92 } \\
\text { - Auguste-Viktoria Krankenhaus, Jerusalem, West-Bank 1993-95 } \\
\text { - Hadassah Universitätsklinik, Jerusalem, Israel 1994-95 } \\
\text { - Abteilung Hämatologie/Onkologie, Philipps-Universität Marburg 1995-96 } \\
\text { - Leitender Oberarzt am Diakoniekrankenhaus, Marburg 1996-2003 } \\
\text { - Seit } 2003 \text { als niedergelassener Facharzt am Tropen-und Reisemedizinischen } \\
\text { Zentrum, Marburg }\end{array}$} \\
\hline $\begin{array}{l}\text { Lehre } \\
\text { - seit } 1996 \mathrm{LE} \\
\text { - } \text { seit } 2005 \mathrm{L \epsilon} \\
\text { Medizin } \\
\text { - } \text { seit } 2003 \mathrm{Fc}\end{array}$ & $\begin{array}{l}\text { trag an der Philipps-Universität Marburg für Tropenmedizin } \\
\text { trag an der Philipps-Universität Marburg für Wilderness- } \\
\text { lungstätigkeit für Ärzte in Reise- und Tropenmedizin }\end{array}$ \\
\hline
\end{tabular}

\section{Zweck der AG-INF}

Die AG-INF stellt den Zusammenschluss von Fachgesellschaften her, die sich mit Themen der Infektionsmedizin in Lehre, Forschung und Praxis befassen.

\section{Die AG-INF}

1. bereitet durch gemeinsame Abstimmung Aussagen und Appelle zu aktuellen Fragen der Infektionsmedizin für die Öffentlichkeit vor

2. fördert die interdisziplinäre Zusammenarbeit der Fachgesellschaften durch gemeinsame Tagungen und verbessert die Effizienz internationaler
Aktivitäten auf dem Gebiet der Infektionsmedizin durch verstärkte Abstimmung innerhalb der Fachgesellschaften

3. wirkt proaktiv und vorausschauend bei der Benennung von Defiziten in der Infektionsmedizin und bei der Definition von Forschungsschwerpunktthemen und unterstützt den Erhalt und den Ausbau universitärer Einrichtungen auf allen Gebieten der Infektionsmedizin

4. wirkt mit an den Konzepten der Fortund Weiterbildungsordnungen in den Bereichen der Infektionsmedizin und der Qualitätssicherung in der Infek- tionsmedizin und arbeitet hier mit Bundes- und Landesärztekammer sowie mit Verbänden und Institutionen der Qualitätssicherung zusammen

5. wirkt mit bei der Entwicklung und Novellierung der Ausbildungskonzepte, des Gegenstandskatalogs und der Approbationsordnung für Studierende der Medizin hinsichtlich infektionsmedizinischer Themen. Darüber hinaus soll sich die AG-INF verstärkt für die Förderung des wissenschaftlichen Nachwuchses in der Infektionsmedizin einsetzen. 